\title{
Lack of interleukin-13 receptor a1 delays the loss of dopaminergic neurons during chronic stress
}

Simone Mori ${ }^{1}$, Shuei Sugama², William Nguyen', Tatiana Michel ${ }^{1,3}$, M. Germana Sanna ${ }^{1}$, Manuel Sanchez-Alavez ${ }^{4}$, Rigo Cintron-Colon', Gianluca Moroncini ${ }^{5}$, Yoshihiko Kakinuma ${ }^{2}$, Pamela Maher ${ }^{6}$ and Bruno Conti ${ }^{1,4,7^{*}}$ (D)

\begin{abstract}
Background: The majority of Parkinson's disease (PD) cases are sporadic and idiopathic suggesting that this neurodegenerative disorder is the result of both environmental and genetic factors. Stress and neuroinflammation are among the factors being investigated for their possible contributions to PD. Experiments in rodents showed that severe chronic stress can reduce the number of dopaminergic neurons in the substantia nigra pars compacta (SNc); the same cells that are lost in PD. These actions are at least in part mediated by increased oxidative stress. Here, we tested the hypothesis that the interleukin-13 receptor alpha 1 (IL-13Ra1), a cytokine receptor whose activation increases the vulnerability of dopaminergic neurons to oxidative damage, participates in the stress-dependent damage of these neurons.
\end{abstract}

Methods: Mice were subject to daily sessions of $8 \mathrm{~h}$ (acute) stress for 16 weeks (5 days a week), a procedure previously showed to induce loss of dopaminergic neurons in the SNc. The source and the kinetics of interleukin-13 (IL-13), the endogenous ligand of IL-13Ral, were evaluated $0,1,3,6$, and $8 \mathrm{~h}$ and at 16 weeks of stress. Identification of IL-13 producing cell-type was performed by immunofluorescent and by in situ hybridization experiments. Markers of oxidative stress, microglia activation, and the number of dopaminergic neurons in IL-13Ra1 knock-out animals (II13ra ${ }^{Y /-}$ ) and their wild-type littermates $\left(\| 13 \mathrm{ra}^{Y /+}\right)$ were evaluated at 16 weeks of stress and at 20 weeks, following a 4 week nonstressed period and compared to non-stressed mice.

Results: IL-13 was expressed in microglial cells within the SN and in a fraction of the tyrosine hydroxylase-positive neurons in the SNc. IL-13 levels were elevated during daily stress and peaked at 6 h. 16 weeks of chronic restraint stress significantly reduced the number of SNc dopaminergic neurons in $1113 \mathrm{ra} 1^{\mathrm{Y} / \mathrm{H}}$ mice. Neuronal loss at 16 weeks was significantly lower in $1113 \mathrm{ra}^{Y /-}$ mice. However, the loss of dopaminergic neurons measured at 20 weeks, after 4 weeks of non-stress following the 16 weeks of stress, was similar in $/ 113 \mathrm{ra}{ }^{Y /+}$ and $/ 113 \mathrm{ra} 1^{Y /-}$ mice.

Conclusions: IL-13, a cytokine previously demonstrated to increase the susceptibility of SNc dopaminergic neurons to oxidative stress, is elevated in the SN by restraint stress. Lack of IL-13Ra1 did not prevent nor halted but delayed neuronal loss in the mouse model of chronic restraint stress. IL-13/LL-13Ra1 may represent a target to reduce the rate of DA neuronal loss that can occur during severe chronic restraint stress.

Keywords: Stress, Interleukin, Parkinson's disease, Neuroinflammation, Microglia, Oxidative stress

\footnotetext{
* Correspondence: bconti@scripps.edu

'Department of Molecular Medicine, The Scripps Research Institute, $10550 \mathrm{~N}$.

Torrey Pines Road, La Jolla, CA 92037, USA

${ }^{4}$ Department of Neuroscience, The Scripps Research Institute, $10550 \mathrm{~N}$.

Torrey Pines Road, La Jolla, CA 92037, USA

Full list of author information is available at the end of the article
} 


\section{Background}

Parkinson's disease (PD) is the second most common neurodegenerative disorder affecting approximately $1 \%$ of the population over the age of 60 [1]. Clinical symptoms include tremors, rigidity, and postural instability which are believed to arise primarily from the progressive reduction of dopamine signaling in the basal ganglia resulting from the loss of dopaminergic (DA) neurons in the substantia nigra pars compacta $(\mathrm{SNc})$. Although several genes have been demonstrated to contribute to familial PD, the majority of PD cases are sporadic and idiopathic, with environmental factors thought to contribute to the development of the disease [2]. Stressful psychological events and neuroinflammation are among the environmental factors being investigated in PD research.

The contribution of stress to neurodegeneration was studied extensively in the hippocampus where the hormone glucocorticoids were demonstrated to mediate neuronal loss [3-5]. Correlative and experimental evidences also suggested that stress may contribute to loss of dopaminergic neurons and thus contribute to the etiology or to the progression of PD [6-14]. Several studies also showed that different types of stressor including restraint, [15-18], prenatal [19], dental disharmony [20], and cold stress [21], can lead to the activation of microglial-increasing reactive oxygen species (ROS) and interleukins [22] creating a neuroinflammatory environment that is believed to contribute to PD [23-25].

In an effort to elucidate the possible contribution of neuroinflammation to PD and how its supposedly nonspecific action could lead to a preferential loss of DA neurons, we previously showed that this is mediated, at least in part, by activation of the receptor alpha 1 of interleukin-13 (IL-13R $\alpha 1$ ) [26]. IL-13R $\alpha 1$ is known for its peripheral role of mediating allergic inflammation and atopy, but it is also expressed centrally in DA neurons of the SNc and the VTA where its activation increased cellular susceptibility to oxidative stress [26]. Interestingly, Il13ra1 is localized at position Xq24 of the human $\mathrm{X}$ chromosome, within a region containing the PARK 12 locus associated with PD.

Preliminary studies showed that restraint stress elevated the level of IL-13 in the CNS [27]. Here, we tested the hypothesis that activation of IL-13R $\alpha 1$ contributes to the loss of DA neurons occurring in a mouse model of severe chronic restraint stress (RS) consisting of daily $8 \mathrm{~h}$ long RS sessions for 5 days a week for 16 weeks [11]. We determined the central cellular source of IL-13 that measured the kinetics of its production and the effects that stress had on microglial activation and oxidative damage during the daily session of $8 \mathrm{~h}$ RS and at 16 weeks. The number of DA neurons in the SNc in mice null for IL-13R $\alpha 1\left(\operatorname{Il13ra1^{Y/-})}\right.$ and their wild-type littermates $\left(\mathrm{Il} 13 \mathrm{ra} \mathrm{I}^{Y /+}\right)$ was determined and compared at 16 or at 20 weeks, 4 weeks after termination of stress.

\section{Methods \\ Animals and tissue harvesting}

Mouse husbandry and procedures were approved and performed under the guidelines of the Institutional Animal Care and Use Committee of The Scripps Research Institute and by the Institutional Animal Care and Use Committee of Nippon Medical School and were performed in accordance with the National Institute of Health Guide for the Care and Use of Laboratory Animals; animal suffering and sacrifice were minimized. All experiments were carried out on 3-6-month-old male mice null for IL-13R $\alpha 1$ (Il13ra1 ${ }^{Y-}$ ) and wild-type littermates $\left(\right.$ Ill3ral $\left.{ }^{Y /+}\right)$ on a $\mathrm{C} 57 \mathrm{BL} / 6$ background [28]. Animals were housed in a room maintained at 20-22 ${ }^{\circ} \mathrm{C}$, on a 12-h light/12-h dark with food and water provided ad libitum. For tissue collection, animals were deeply anaesthetized using 4-5\% isoflurane and subsequently perfused with $0.1 \mathrm{M}$ phosphate buffer for real-time PCR analysis or with $0.1 \mathrm{M}$ phosphate buffer followed by $4 \%$ paraformaldehyde (PFA) for histological analysis. After overnight post-fixation in 4\% PFA, the brains to be used for histological analysis were cryoprotected in 30\% sucrose then frozen at $-80{ }^{\circ} \mathrm{C}$. The brains were sectioned using a Leica cryostat to produce $35 \mu \mathrm{m}$ slices for both immunohistochemistry and immunofluorescence.

\section{Stress experiment}

Restraint stress (RS) was performed as previously described [11] on age-matched Il13ra1 ${ }^{Y /-}$ and $I l 13 \mathrm{ra1}{ }^{Y /+}$ mice. Briefly, animals were placed in ventilated plexiglass restrainers that prevented the mice from turning without causing any pain for a maximum of eight consecutive hours per day. Chronic stress was achieved by performing this acute stress procedure daily for 5 days a week for a maximum of 16 weeks; a regimen previously shown by us to induce loss of DA neurons in the SNc [11] (Fig. 1a, b). Tissues were also collected at the end of the last 8 -h session at 16 weeks RS or at the same time of day after 4 additional weeks after termination of stress (week 20) (Fig. 1c).

\section{Immunohistochemistry}

Immunofluorescence microscopy was performed on $35-\mu \mathrm{m}$ brain slices containing the region of interest (see Additional file 1: Figure S1a). The following primary antibodies were used: rabbit-anti Iba-1 (1:500, Wako); rabbit anti-GFAP (1:250, Thermo Fisher); mouse monoclonal anti-NeuN (1:250, Millipore); goat anti-IL-13 (1:50, R\&D system), rabbit anti-3-Nitrotyrosine (3-NT, 1:200, Millipore). After washing, a combination of secondary antibodies was used for detection: 488 Alexa goat anti-rabbit, 594 Alexa goat anti-mouse IgG1 (Invitrogen). Slides were 


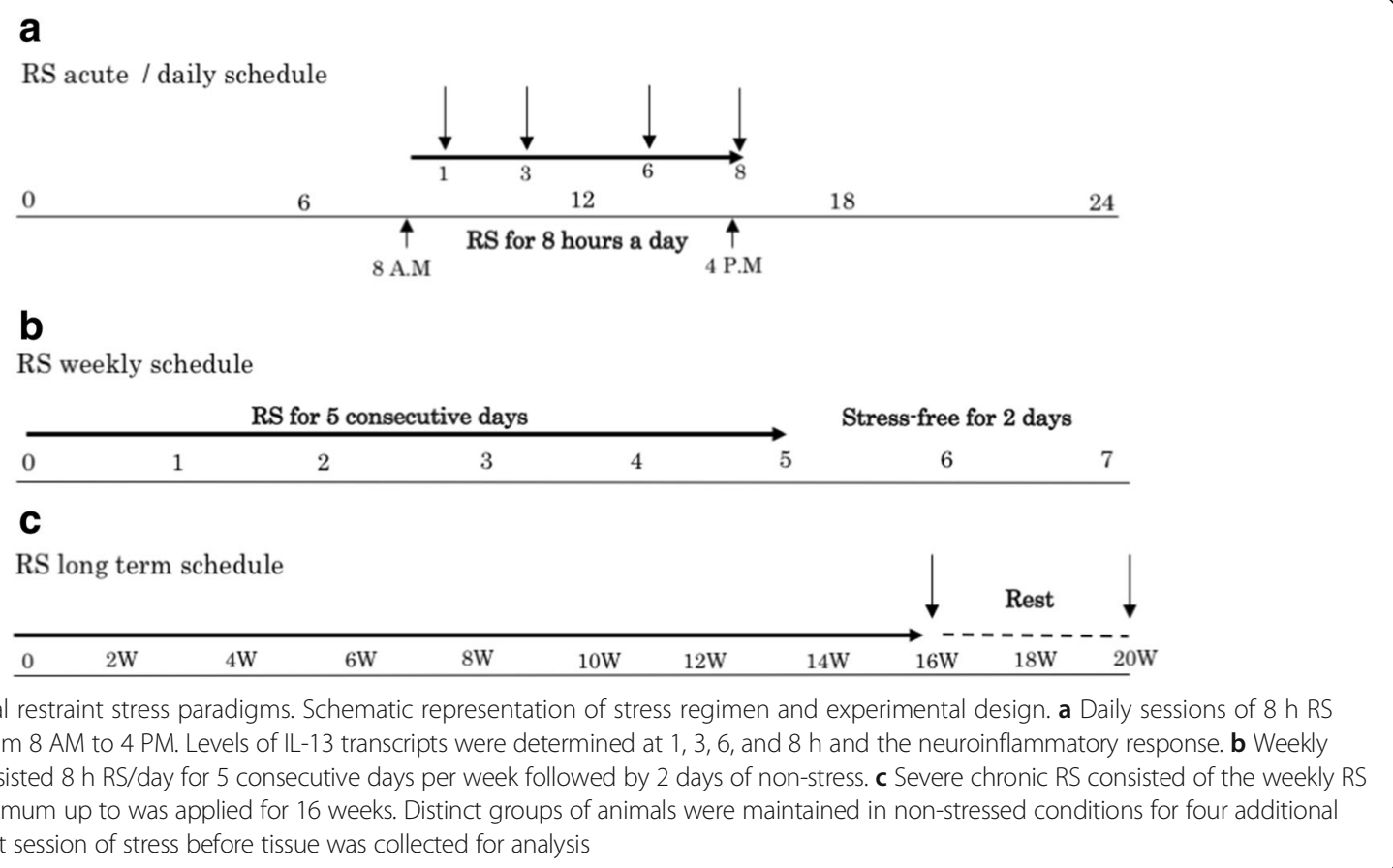

counterstained with DAPI and were cover-slipped in Fluoromount. Digital images were taken using a Zeiss LSM 710 laser scanning confocal microscope (LSCM). Tyrosine Hydroxylase DAB Immunohistochemical analysis was performed using rabbit anti-Tyrosine Hydroxylase, (1:10000; Millipore), incubated with a biotinylated goat-anti rabbit antibody (1:400; Vector Labs) for $1 \mathrm{~h}$ at RT and then stained with Vectastain ABC Kit (Standard) and DAB Peroxidase Substrate (Vector Labs). For cresyl violet (CV) staining (Nissl), adjacent sections were mounted on positive charged slides and air-dried. The slides were soaked in CV solution ( $0.25 \%$ cresyl violet and $0.3 \%$ acetic acid) for $15 \mathrm{~min}$ (warmed at $50{ }^{\circ} \mathrm{C}$ ), were dehydrated with alcohol and xylene, and were cover-slipped with Vectamount.

\section{Cell counting}

Cell counting was performed by unbiased stereology. Briefly, digital images of $\mathrm{TH}$ - and $\mathrm{CV}$-positive cells were acquired on the SNc at $\times 50$ magnification on an Olympus microscope fitted with a video camera (Olympus, Tokyo, Japan). Counting frames $(100 \times 100 \mu \mathrm{m})$ were generated using MCID image analysis software (Imaging Research Inc., Ontario, Canada) and were systemically scanned over the outlined SNc using a motorized stage. Neurons were counted when they appeared within a square $(50 \times 50 \mu \mathrm{m})$ of the counting frame but were not in contact with the left or bottom border. This procedure was carried out on every four sections at a periodicity of $140 \mu \mathrm{m}$ through the SNc. Finally, total SNc neuron number was calculated as the product of the neuron density and the volume of the SNc as previously reported $[29,30]$. To count the number of double-positive-labeled cells (IL13-green) and (Iba-1, GFAP, NeuN, or Tyrosine Hydroxilase-red), we used region of interest analysis macros in Image Pro Premier (Media Cybernetics) [28]. Digital images were taken using a Zeiss LSM 710 laser scanning confocal microscope LSCM. A maximum intensity projection (MIP) was created of each multi-sectioned reconstruction of the doublepositive-labeled cells using the Zen software. For further processing, the MIPs of each sample were imported into Image Pro Premier (Media Cybernetics) where the region of the SN was defined (outline) to create a mask of the whole region of interest. Four counting frames $(100 \times$ $100 \mu \mathrm{m})$ were generated using Image Pro Premier Software for each MIP image. The number of red, green, and total nuclear count (DAPI) in each frame was counted with the exclusion of those not in contact with the left or bottom border. These results were represented as number of cell/total nuclear count extracted from the total area for each fluorescent-labeled population. Double-positive populations were defined by scoring, outlined as a region of interest, all of one population (signal Red-TH), and obtaining from the software how much of the other (green-IL-13) resides within the red-positive region of interest. 3-NT fluorescence intensity arbitrary units (a.u.) were calculated by dividing the detected optical density by area of the region of interest.

\section{Tissue processing for protein quantification and semi-quantitative PCR}

Real-time PCR and IL-13 ELISA were performed on SN samples from IL-13R $\alpha 1$ wild-type mice. Animals (6-7 
per group) were perfused intracardially with PBS at different time points of acute RS $(3,6$, and $8 \mathrm{~h})$, and the SN was rapidly dissected and flash frozen. Total RNA was isolated using a RNA extraction kit (NucleoSpin RNA Plus, Macherey-Nagel \#740984.250) according to the manufacturer's protocol. After eluting the RNA product with RNase/DNase-free water, the concentration and purity of the RNA was determined using a NanoDrop ND-1000 spectrophotometer. Total RNA was reverse transcribed into cDNA using a reverse transcription cDNA synthesis kit (Maxima cDNA Synthesis Kit, Thermo Fisher, \#FERK1642) per the manufacturer's protocol. The cDNA templates were amplified via PCR using the Taq polymerase enzyme (PowerUP Sybr Master Mix, Life Technologies, \#A25777) along with mouse IL-13specific primers obtained from Qiagen RT2 qPCR Primer Assay, (PPM03021B). IL-13R $\alpha 1$ primers used as follows: FW 5 ' -cct tgc ttc tgt ggt agt aga g-3' and RW 5' - aaa ggg cca gga gga aat ac-3'. A Bio-Rad CFX 384 PCR System was used to perform the qPCR reactions. Data were analyzed using the $\Delta \Delta \mathrm{Ct}$ method with the levels of the gene of interest normalized to the beta Actin housekeeping gene. For protein extraction, SN samples were homogenized in $400 \mu \mathrm{l}$ of lysis buffer $\left(\mathrm{N}-\mathrm{PER}^{\mathrm{TM}}\right.$ Neuronal Protein Extraction Reagent, Thermo Fisher, plus 1 protease inhibitor cocktail tablet) and then were centrifuged at $14,000 \mathrm{~g}$ for $30 \mathrm{~min}$ at $4{ }^{\circ} \mathrm{C}$. The supernatants were collected, and protein concentrations were determined by Pierce BCA Protein Assay Kit (Thermo Fisher Scientific, USA). Samples were analyzed in a ELISA for mouse IL-13 (R\&D) according to the manufacturer's instruction, and results normalized for total protein (pg IL-13/mg total protein).

\section{Statistical analysis}

One-factor ANOVA followed by a Bonferroni post hoc test was used to determine statistical significance $\left({ }^{*} p<0.05,{ }^{* *} p<0.01 ; * * * 0<0.001\right)$ using GraphPad Prism Software (La Jolla, CA, USA).

\section{Results}

IL-13 is increased by acute RS in the substantia nigra

IL-13R $\alpha 1$ is expressed in dopaminergic neurons where its activation during neuroinflammation increases their vulnerability to oxidative stress in the SNc [26]. We previously demonstrated that repetitive sessions of acute daily of $8 \mathrm{~h}$ RS can eventually reduce the number of DA neurons in the SNc [11]. To determine whether the daily session of acute stress would modify the level of IL-13, we measured IL-13 mRNA, immunoreactivity, and protein level in the $\mathrm{SN}$ of animals restrained for $0,1,3,6$, or $8 \mathrm{~h}$ (Fig. 2). Semi-quantitative RT-PCR analysis revealed that RS elevated the IL-13 mRNA. The increase was the highest and most significant at $6 \mathrm{~h}$ of RS with fold change compared to non-stressed animals being
$3.89 \pm 1.545, n=6, p=0.04$. Fold difference compared to control (arbitrarily assigned the value of 1 ) did not reach statistical significance at the other time-point tested $(0.37 \pm 0.032, n=6, p>0.99$ at $1 \mathrm{~h}$ RS; $0.90 \pm 0.186, n=6$, $p>0.99$ at $3 \mathrm{~h} \mathrm{RS} ; 2.63 \pm 0.704, p=0.74$ at $8 \mathrm{~h} \mathrm{RS})$ (Fig. 2a). The levels of IL-13R $\alpha 1$ mRNA were also determined (Fig. 2b) with no alterations on the mRNA levels of the IL-13 receptor at all time points. ELISA demonstrated that RS also elevated the level of IL-13 protein and that such increase was statistically significant at $8 \mathrm{~h}$ when the fold change compared to non-stressed animals was of a similar order of magnitude as the transcript at $6 \mathrm{~h}$ (IL-13 in pg/mg total protein: $2.644 \pm 0.50$ control; $5.607 \pm 2.793$ at $1 \mathrm{~h}$ RS, NS; $7.128 \pm 3.123$, at $3 \mathrm{~h}$, NS; $8.857 \pm 1.361$ at $6 \mathrm{~h} \mathrm{RS}$, NS, and $11.7 \pm 3.195$ at $8 \mathrm{~h}$ RS, $p<0.05$ ) (Fig. 2c).

Immunohistochemistry further confirmed that IL-13 protein expression increased at $8 \mathrm{~h}$ of RS and demonstrated that the cellular source of IL-13 was distributed in both the $\mathrm{SN}$ pars compacta $(\mathrm{SNc})$ and the $\mathrm{SN}$ pars reticulata $(\mathrm{SNr})$ (Fig. 3A-A'). Similar results were obtained when evaluating the presence and distribution of IL-13 by immunohistochemistry at 16 weeks of stress (Fig. 3A' $\mathrm{A}^{\prime \prime}$. These data indicate that stress paradigm used here elevated IL-13 providing evidence that the treatment used effectively led to a local increase of IL$13 R \alpha 1$ ligand.

\section{In the substantia nigra IL-13 is produced by microglia and neurons}

The source of IL-13 in the SN after acute and long-term RS exposure was determined by dual immunohistochemistry staining as well as by a combination of in situ hybridization/immunohistochemistry (Fig. 3 and Additional file 1: Figure $\left.\mathrm{S} 1 \mathrm{C}-\mathrm{C}^{\prime \prime}\right)$. Double immunofluorescence was performed for IL-13 and one of the following: the microglial marker Iba-1, the astrocytic marker GFAP, the pan-neuronal marker NeuN, or the catecholaminergic neuronal marker tyrosine hydroxylase (TH). IL-13 was expressed in the SNr and in part of the SNc (Fig. 3a) and co-localized with Iba1 as well as with NeuN (Fig. 3B-B' ', D-D' ') but not with GFAP (Fig. 3C$\left.\mathrm{C}^{\prime \prime}\right)$. IL-13 was also found in a fraction of the TH-positive neurons in the $\mathrm{SNc}$ (Fig. 3E'-E' '). Following acute RS, microglia accounted for approximately 77\% (4123 \pm 204 Iba-1-positive cells out of $6450 \pm 444$ IL-13-positive cells) of the IL-13-positive cells. NeuN- and TH-positive cells accounted for 14 and $7 \%$, respectively, of the remaining IL-13-positive cells. Similar values were detected after chronic stress exposure (Iba-1: $82 \%$, NeuN: $13 \%$, and TH: 4\%), (see Additional file 1: Fig. S1B). Neuronal expression of IL-13 was also confirmed by double in situ hybridization/immunofluorescence for IL-13 mRNA and NeuN, respectively (see Additional file 1: Figure S1C-C"). 

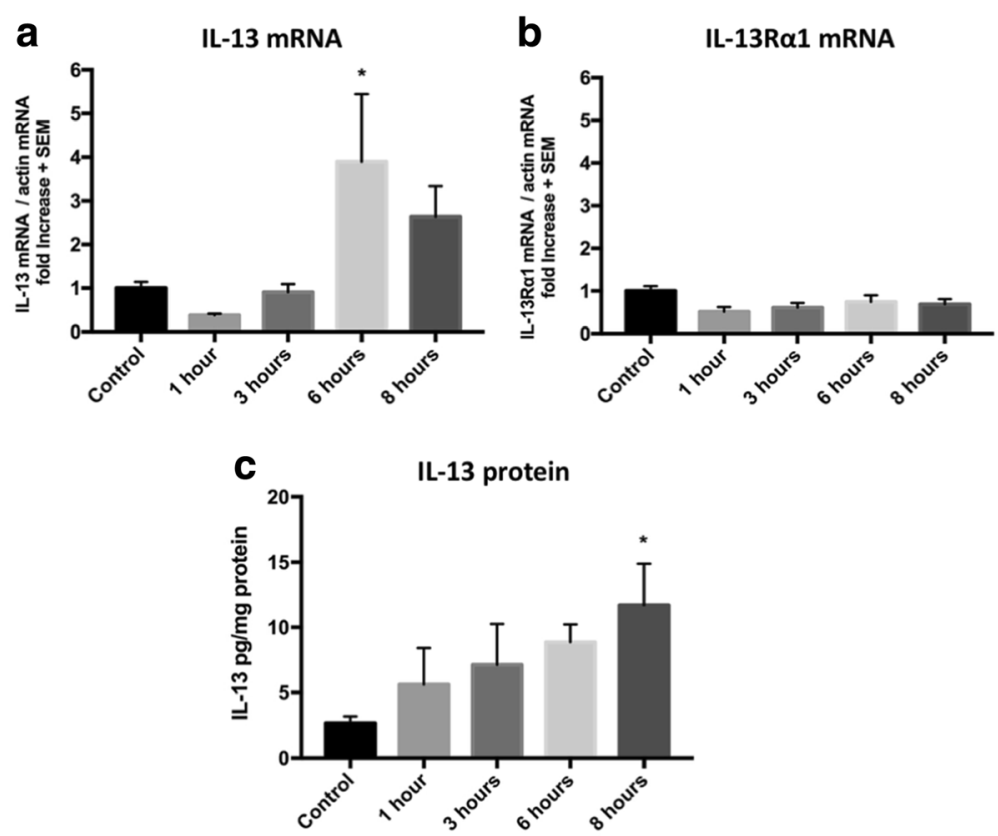

Fig. 2 Acute restraint stress induces central production of IL-13 in the substantia nigra. a Semi-quantitative RT-PCR performed on RNA extracted from the SN of mice treated with acute RS showed that compared with the level of control animals, arbitrarily fixed to $1(n=8)$, levels were 0.37 $\pm 0.032, p>0.99 ; 0.90 \pm 0.186, p>0.99 ; 3.89 \pm 1.545, p=0.04$ and $2.63 \pm 0.704, p=0.74$ at $1,3,6$, and 8 h, respectively, $n=6$. b Semi-quantitative RT-PCR assessing expression of IL-13Ra1 on same samples described in figure A shows no difference in all time points compared to control condition ( $p>0.99$ at control, 1, 3, 6, and 8 h). c Protein quantification in the SN shows upregulation of IL-13 during restraint stress (IL-13 in pg/mg total protein: $2.644 \pm 0.50$ control; $5.607 \pm 2.793$ at $1 \mathrm{~h} \mathrm{RS}, p>0.99 ; 7.128 \pm 3.123$, at $3 \mathrm{~h}, p=0.679 ; 8.857 \pm 1.361$ at $6 \mathrm{~h} \mathrm{RS}, p=0.1612$, and $11.7 \pm 3.195$ at $8 \mathrm{~h} \mathrm{RS}, p=0.0342$ )

Oxidative stress and microglia activation during restraint stress are similar in $/ 113 \mathrm{ra} 1^{\mathrm{Y/}-}$ and $\| 13 \mathrm{ra}^{\mathrm{Y/+}}$ mice

Activation of IL-13R $\alpha 1$ contributes to neurotoxicity by increasing cellular susceptibility to oxidative stress [26]. We measured 3-Nitrotyrosine (3-NT) as an index of oxidation (Fig. 4a, b). We found that 3-NT levels were similar in both Il13ral $^{Y /-}$ and Il13ra1 ${ }^{Y /+}$ non-stressed mice $(9.42 \pm 0.86$ a.u. vs. $10.22 \pm 0.23$ a.u., $n=4$, NS) and were increased similarly and significantly $(p<0.001)$ and in both groups following $8 \mathrm{~h}$ of RS $(21.23 \pm 2.24$ a.u. vs. $19.89 \pm 1.36$ a.u.; $n=4-6$, NS). At 16 weeks of RS, 3-NT levels across genotypes were still significantly higher than in non-stressed animals used as controls but did not differ statistically in Il13ra1 ${ }^{Y /-}$ compared to Il13ra1 ${ }^{Y /+}$ mice $(16.86 \pm 2.18$ a.u. vs. $20.52 \pm 2.91$ a.u., $p=0.8)$. 3-NT levels at 20 weeks, 4 weeks after termination of stress, were similar to those of non-stressed controls animals and were not statistically different across genotypes (10.29 \pm 2.18 a.u. vs. $10.43 \pm 0.14$ a.u., $n=4$, NS). These data indicate that RS increases oxidative stress similarly in both genotypes demonstrating that ROS required for IL-13 toxicity were equally present in $I l 13 \mathrm{ral}^{Y /-}$ and $\mathrm{Il13ra1^{Y/+ }}$ mice.

Evaluation of the microglial marker Iba-1 at the same time points in $I l 13 \mathrm{ral}^{Y /-}$ and $I l 13 \mathrm{ra1} 1^{Y /+}$ mice showed that RS increase microglia activation similarly in knock- out and wild-type animals (Fig. 4c, d). Iba-1 immunoreactivity was significantly increased in for $I l 13 \mathrm{ral}^{Y /-}$ mice and their wild-littermates after acute and chronic RS $\left(p<0.05\right.$ at $8 \mathrm{~h}$ and 16 weeks for Il13ral $^{Y /-}, p<0.05$ at $8 \mathrm{~h}$, and $p<0.001$ at 16 weeks for $I l 13 \mathrm{ral}^{Y /+}$ ) and was lowered after 4 weeks of non-stressed condition (NS all groups). No statistical difference was detected at any time point between genotypes. These data confirm previous finding that RS induce neuroinflammation in the $\mathrm{SN}$ of wild-type animals and indicate that Ill3ra1 $1^{Y /-}$ mice present the same level of microglial activation response after RS.

\section{Lack of interleukin-13 receptor a1 delays the loss of dopaminergic neurons during chronic stress}

We next evaluated whether RS affected similarly or differently the number of DA neurons in $I l 13 \mathrm{ra} 1^{Y /-}$ and Il13ra1 $1^{Y /+}$ mice (Fig. 5a, b). Non-stressed Il13ra1 ${ }^{Y /-}$ and Il13ra1 $1^{Y /+}$ mice had similar numbers of $\mathrm{TH}$-positive neurons in the SNc $\left(9405 \pm 231\right.$ for $I l 13 r a 1^{Y /+}$ mice vs. $9240 \pm 127$ for $I l 13 \mathrm{ra1}^{Y /-}$ mice, $n=4$, NS), and these numbers were found to be similar following a single acute RS session $\left(9556 \pm 113\right.$ for Il13ra1 $^{Y /+}$ mice vs. $9346 \pm 176$ for Il13ra1 ${ }^{Y /-}$ mice, $n=4$, NS when compared to non-stressed control). Chronic RS reduced the number of $\mathrm{TH}$-positive neurons in both wild-type 


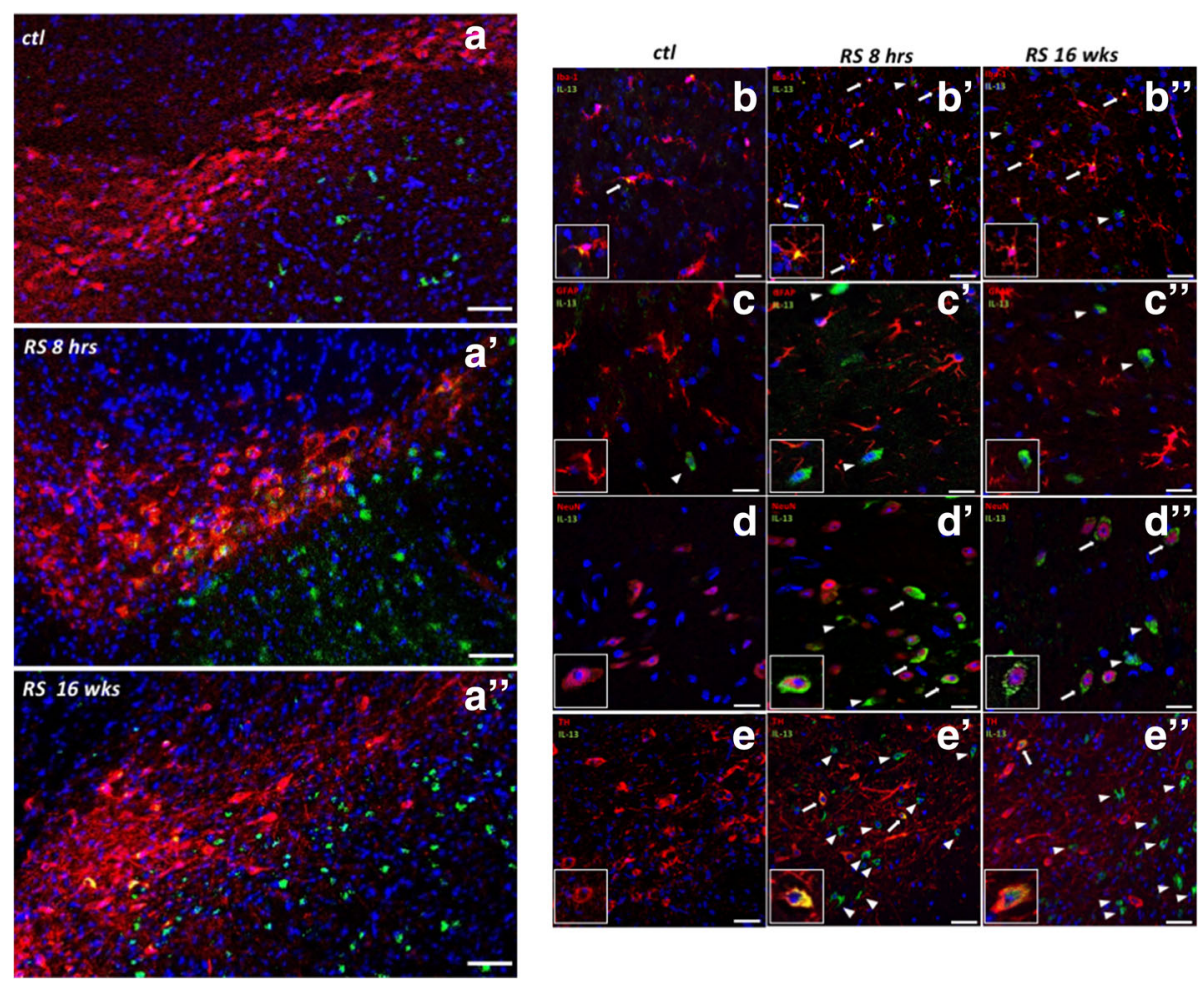

Fig. 3 Mapping the location of IL-13 production in the substantia nigra. (A-A") Representative pictures of immunofluorescent staining of IL-13 (green) in the SN of non-stressed (top), $8 \mathrm{~h}$ of RS (center) and after 16 weeks of RS (bottom); showing that RS elevates IL-13 expression in both the SNc and the SNr. (Pictures representative of a $n=3$ experiment; scale bars: 0.5 mm; blue: DAPI). (B-B", C-C", D-D", E-E") Representative pictures of double immunofluorescence of IL-13 (green) with Iba-1, GFAP, NeuN, and TH (red), respectively, in control condition, 8 h of RS, and 16 weeks of RS. Arrows: co-localization of IL-13 and the specific cell type marker; arrowheads: IL-13 signal without co-localization with the cell type marker. IL-13 co-localizes (arrows) with microglia (Iba-1, Fig. B-B"), neurons (NeuN, Fig. D-D"), and dopaminergic cells (TH, Fig. E-E"). No co-staining was found in astrocytes (GFAP, Fig. C-C"). (scale bar: $200 \mu \mathrm{m}$ in $\mathbf{A}-\mathbf{A}^{\prime \prime}, 50 \mu \mathrm{m}$ in $\mathbf{B}-\mathbf{E}^{\prime \prime}$; blue: DAPI)

Il13ra1 ${ }^{Y /+}(9405 \pm 231$ for control vs. $4180 \pm 1001$ for RS mice at 16 weeks, $n=4, p<0.0001)$ and $I l 13 \mathrm{ra} 1^{Y /-}$ mice, $(9240 \pm 127$ for control vs. $6490 \pm 404$ for RS mice, $n=4, p$ $<0.01)$. However, Il13ra1 ${ }^{Y /-}$ mice retained a significantly larger number of neurons than their wild-type littermates did (6490 \pm 404 vs. $4180 \pm 1001$ for RS mice, $n=4, p<0.05)$ indicating that $I l 13 \mathrm{raI}^{Y /-}$ mice are partially protected from the loss of DA neurons during chronic RS.

Measurements were also carried out on groups of animals that underwent the same stress paradigm but were analyzed following 4 weeks of rest after the 16 weeks of RS to evaluate whether the stress-dependent reduction in the number of TH-immunopositive cells would halt or continue. Data from these groups showed that the number of TH-positive neurons in the $I l 13 \mathrm{ra} 1^{Y /+}$ mice remained comparable to that observed at 16 weeks while the number of $\mathrm{TH}$-positive neurons in the $I l 13 \mathrm{ral}^{\mathrm{Y}-}$ mice further decreased to reach a value similar to that in the $I l 13 \mathrm{ral}^{Y /+}$ mice $\left(4647 \pm 144\right.$ for $I l 13 \mathrm{ra} 1^{Y /+}$ mice vs. $5335 \pm 239$ for $I l 13 \mathrm{ral}^{Y /-}, n=4$, NS). These data indicate that lack of IL-13R $\alpha 1$ did not halt but rather delayed neuronal loss during RS. To ensure that the observed loss of $\mathrm{TH}$ immunoreactivity reflected cellular death rather than downregulation of $\mathrm{TH}$ expression, cell numbers were evaluated using cresyl violet staining on adjacent sections (Fig. 5c, d). We confirmed that the reduction of cells in the $\mathrm{SN}$ develops during chronic restraint stress in wild-type animals $(p<0.05$ at 16 weeks and 16 weeks + rest compared to control) and is delayed in $I l 13 \mathrm{ral}^{Y /-}$ mice $(p<0.05$ at 16 weeks compared to Il13ra1 ${ }^{Y /+}$ animals) but not completely halted where a statistical significant reduction was also found $(p<0.05$ at 16 weeks + rest compared to control).

\section{Discussion}

In this study, we tested the hypothesis that IL-13R $\alpha 1$ is involved in the loss of DA neurons that is observed in a mouse model of severe chronic stress where animals are exposed to daily $8 \mathrm{~h}$ session of restraints stress for 5 consecutive days per week to a total of 16 weeks [11].

IL-13R $\alpha 1$ is expressed on DA neurons of the SNc, and its activation was previously shown to increase the susceptibility of these cells to oxidative stress [26]. We found that the daily RS session elevated IL-13 in the 


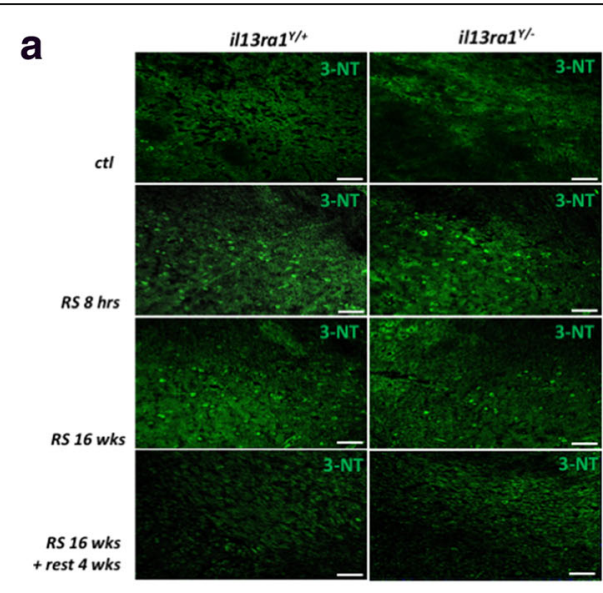

C

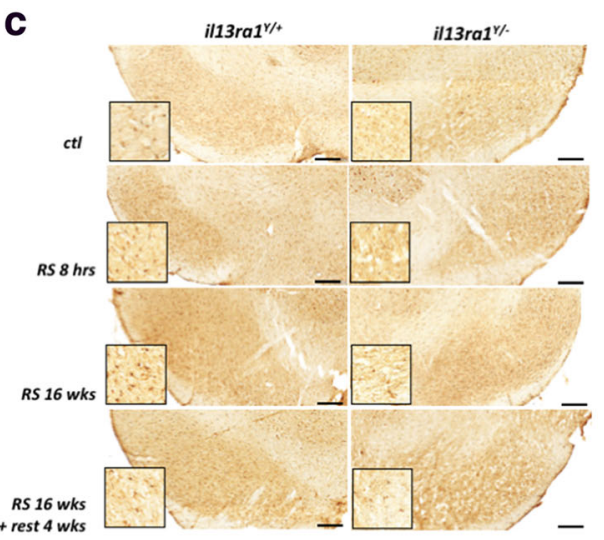

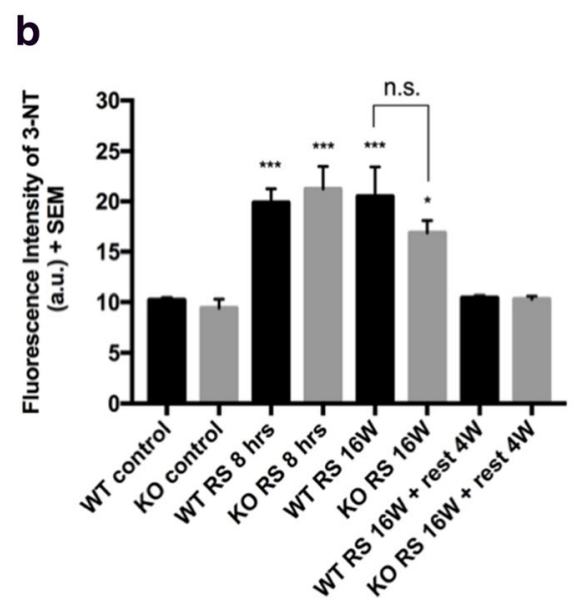

d

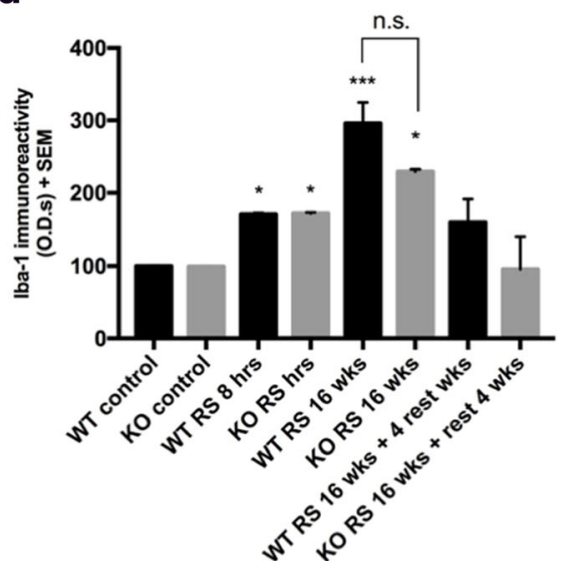

Fig. 4 IL-13Ra1 does not alter restraint stress-associated neuroinflammation. a Representative images showing immunofluorescence of 3-Nitrotyrosine (3-NT, green) in the SN under resting conditions and at different paradigms of RS in IL-13Ra1 wild-type and knock-out animals. Levels of oxidative damage were increased during restraint stress with no difference between genotypes. $\mathbf{b}$ Histograms showing the average levels of fluorescence intensity (in a.u.) of 3-Nitrotyrosine fluorescence in the SN. c Representative pictures of microglial marker lba-1 under resting conditions and at different paradigms of RS in IL-13Ra1 wild-type and knock-out animals. Levels of oxidative were increased during restraint stress with no difference between genotypes. $\mathbf{d}$ Histograms showing the average levels of O.D.s (in a.u.) of Iba-1 in the SN ( ${ }^{*} p<0.05,{ }^{* *} p<0.001$ compared to control condition, $n=4-6$; scale bars: $100 \mu \mathrm{m}$ in $\mathrm{A}, 200 \mu \mathrm{m}$ in $\mathbf{C}$

substantia nigra, stimulated microglia activation, and elevated oxidative stress which is necessary for IL-13mediated toxicity. We also collected evidence that IL-13 is produced in both the pars compacta $(\mathrm{SNc})$ and the pars reticularis $(\mathrm{SNr})$ regions of the substantia nigra, and that cellular sources comprises microglia and neurons, including a fraction of TH-positive ones. Neuronal expression of IL-13 was previously reported in the hippocampus and the cortex following an experimental ischemic insult [31, 32], and recent studies suggested a role of this cytokine in cognitive functions [33]. Our findings showed that stress can regulate IL-13 production locally and suggest the intriguing possibility that it may act in a paracrine or, in the case of $\mathrm{TH}$ neuron, possibly even in an autocrine fashion. We previously demonstrated that activation of IL-13R $\alpha 1$ alone did not damage DA neurons indicating that the IL-13/IL-13R $\alpha 1$ system is important for the physiology of dopaminergic cells [26]. Indeed, IL-13 was found to be toxic to dopaminergic cells of the SNc only in the presence of mild oxidative stress and, until now, only in experimental paradigms employing chronic prolonged insult like regular intermittent exposure to LPS for 6 months [26].

These features make the IL-13/IL-13R $\alpha 1$ a particularly attractive subject of investigation in the etiology of PD, a neurodegenerative disease believed to develop and progress over a long period of time. For the same reason, we hypothesized that IL-13/IL-13R $\alpha 1$ could have a role in the loss of dopaminergic neurons suggested to occur during chronic stress $[7,9,11-13,15,34-39]$.

We previously showed the RS paradigm employed here (which is particularly severe and longer compared 

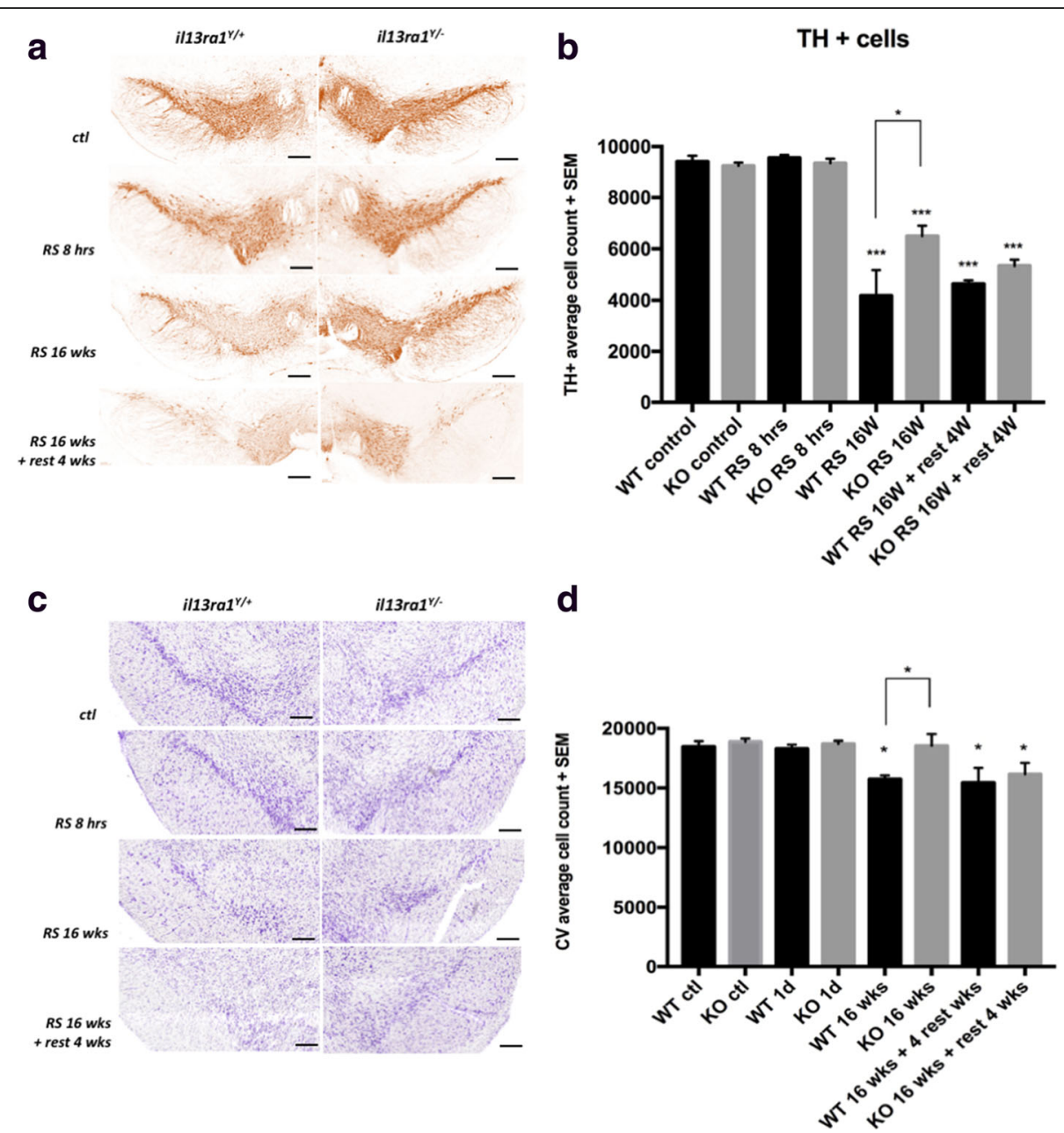

Fig. 5 IL-13Ra1 contributes to dopaminergic neuronal loss during chronic stress. a Representative images of labeling of TH+ neurons using wildtype $/ 113 \mathrm{ra}^{Y /+}$ and knock-out $/ 113 \mathrm{ra}^{Y /-}$ mouse brains from control mice and at different time points in the RS paradigm. $\mathbf{b}$ Histograms showing the number of TH+ neurons in the $1 / 13 \mathrm{ra}^{Y /+}$ and $/ 113 \mathrm{ra}^{Y /-}$ in the SNc under control conditions, RS $8 \mathrm{~h}$, after 16 weeks of RS, and after 16 weeks of RS plus 4 weeks of rest. c Representative images of cresyl violet labeling (Nissl staining) cells used in adjacent section to the ones assessed by TH staining. $\mathbf{d}$ Histograms showing the average cell count of $\mathrm{CV}+$ cells in the $/ 113 \mathrm{ra}^{Y /+}$ and $/ 113 \mathrm{ra}{ }^{Y /-}$ in the SNc under control conditions, RS $8 \mathrm{~h}$, after 16 weeks of RS, and after 16 weeks of RS plus 4 weeks of rest. ( ${ }^{*} p<0.05$, ${ }^{* *} p<0.001$ compared to control condition, $n=4-6 ;$ scale bars: $200 \mu \mathrm{m}$ in $\mathbf{A}$, C)

to other studies [40-43]) led to a $61 \%$ reduction in $\mathrm{TH}-$ positive neurons by 16 weeks of treatment [11], a finding recently replicated by an independent group using a similar paradigm [14]. Here, we further confirm the deleterious effects of chronic stress on DA neurons of the SNc as $I l 13 \mathrm{ral}^{Y /+}$ mice lost $56 \%$ of DA neurons in the SNc. We also found that mice lacking a functional IL-13R $\alpha 1$ lost only $30 \%$ of cell. This, together with the evidence that the endogenous IL-13R $\alpha 1$ ligand IL-13 level in the SN is elevated by restraint stress, suggests that IL-13 and IL-13R $\alpha 1$ play a role in mediating the damaging effects of chronic stress on DA neurons. Stress induced oxidative damage and microglia activation, two possible mediators of neuronal damage, were affected similarly in $I l 13 \mathrm{ra}^{Y /-}$ and $I l 13 \mathrm{ra} 1^{Y /+}$ mice indicating that IL-13R $\alpha 1$ did not alter these parameters. Therefore, it seems reasonable to conclude that one of the mechanisms accounting for the reduction in dopaminergic cell loss in the Il13ra $1^{Y /-}$ mice include the lack of the synergistic action between neuronal IL-13R $\alpha 1$ and oxidative stress that was previously shown to exert toxicity in DA cell line [26].

In this study, we also addressed whether the damaging effects of chronic stress on dopaminergic neurons of the $\mathrm{SNc}$ continued upon termination of stress or not. We found that 4 weeks after stress was terminated, and the number of $\mathrm{TH}$-positive neurons was similar to those measured at the termination of stress in $\mathrm{Il13ra}^{Y /+}$ but further decreased in $I l 13 \mathrm{ral} 1^{Y /-}$ to a number that was still higher but not statistically significant than that of wildtype mice. This indicates that the lack of IL-13R $\alpha 1$ did not halt but rather delayed neuronal loss. The 
mechanisms accounting for such phenomenon remain to be determined. One possibility is that activation of IL$13 R \alpha 1$ is only one of the factors contributing to the stress-dependent loss of these neurons. Another is that the stress paradigm utilized was so severe that the free radical damage caused develops over time to a level that is sufficiently large to damage even neurons lacking IL$13 R \alpha 1$ overriding the neurotoxicity that is observed when both IL-13 and sub-lethal doses of reactive oxygen species are present. The use of stress as an experimental paradigm to investigate loss of DA neurons is at its infancy and it will be important to investigate the effects of shorter or milder stressors.

\section{Conclusions}

We confirmed that severe chronic restraint stress can reduce the number of DA neurons and that the IL-13/IL$13 R \alpha 1$ system is one, albeit not the only factor, contributing to these effects. We also showed that restraint stress might act as trigger of central production of IL-13. Blocking IL-13 or IL-13R $\alpha 1$ may contribute to delay damage to DA neurons occurring during chronic severe stress.

\section{Additional files}

Additional file 1: Methods: in situ hybridization. In situ hybridization was carried out as previously described [44] with fluorescent/peroxidase (POD) conjugate staining for IL-13. Anti-sense and sense digoxigenin (DIG)-labeled IL-13 riboprobes were synthesized using a commercial kit (Roche, Indianapolis, IN, USA) from a plasmid (pcr2.1-TOPO) containing full-length IL-13 CDNA (5'-CTT GCC TTG GTG GTC TCG-3', 5'-CGT TGC ACA GGG GAG TCT-3'). Prehybridization and hybridization were then performed at $65{ }^{\circ} \mathrm{C}$ in a buffer containing $50 \%$ formamide, $2 \times$ SSC, $5 \times$ Denhardt's reagent, $5 \%$ Dextrane sulfate, $0.5 \mathrm{mg} / \mathrm{ml}$ sheared salmon sperm DNA, and $0.25 \mathrm{mg} / \mathrm{ml}$ yeast total RNA. The probe was diluted in the hybridization buffer ( $800 \mathrm{ng}$ / $\mathrm{ml}$ ) and was incubated overnight on slides. After post-hybridization washes, slides were then blocked for $1 \mathrm{~h}$ and were incubated with anti-DigoxigeninPOD (11207733910 Roche), 1:1000 overnight at $4^{\circ} \mathrm{C}$. After several rinses in PBS-T, reactions were developed with TSA Plus Fluorescein substrate (NEL741001KT) for $10 \mathrm{~min}$. Sections were then rinsed and were cover-slipped. Digital images were taken using a Zeiss LSM 710 laser scanning confocal microscope (LSCM). Figure S1 (A) Representative pictures of the region of interested evaluated in the cellular counting (substantia nigra, from -2.7 to $-3.8 \mathrm{~mm}$ from bregma). (B) Graph showing double-labeled cells positive for IL-13 and for Iba-1, NeuN, or TH $\left(n=4,{ }^{* *} p<0.01\right.$ compared to control). $\left(C-C^{\prime \prime}\right)$ In situ hybridization of IL-13 mRNA using anti-sense RNA probe in representative wt $\left(/ / 13 \mathrm{ra}^{Y /+}\right)$ confirms co-localization with neuronal marker NeuN (arrows). Analysis with IL-13 sense probe yields no detectable signal (not shown). (Pictures representative of a $n=3$ experiment, scale bars: $20 \mu \mathrm{m}$ in $\mathrm{G}-\mathrm{G}^{\prime \prime}$, blue: DAPI). (TIFF $5303 \mathrm{~kb}$ )

\footnotetext{
Abbreviations

3-NT: 3-Nitrotyrosine; CNS: Central nervous system; DA: Dopaminergic; IL-13: Interleukin-13; IL-13Ra1: Interleukin-13 receptor alpha 1; LPS: Lipopolysaccharide; LSCM: Laser scanning confocal microscope; MPTP: 1-methyl-4-phenyl-1,2,3,6-tetrahydropyridine; PD: Parkinson's disease; ROS: Reactive oxygen species; RS: Restraint stress; SN: Substantia nigra; SNc: Substantia nigra pars compacta; TH: Tyrosine hydroxylase
}

\section{Acknowledgements}

The authors thank Shyam Srinivasan, Ph.D. (Salk Institute), William Kiosses, Ph.D. (TSRI), and Kathy Spencer, Ph.D. (TSRI) for their support and assistance in stereology methods and confocal microscopy.

\section{Funding}

Funding Supported by the NIH (NS085155), The Michael J. Fox Foundation, the College of Physician and Surgeons of Ancona, Italy and The Luxembourg National Research Fund INTER/MOBILITY/2015/10533315/IMMUNPARK.

\section{Availability of data and materials}

All data generated or analyzed during this study are included in this published article. Supplementary information files are available from the corresponding author on reasonable request.

\section{Authors' contributions}

SM, SS, YK, and BC conceived the project and wrote the paper. SM, SS, WN, TM, MGS, MS-A, and RC-C performed the experiments. SM, SS, YK, and BC analyzed and interpreted the data. GM and PM contributed in the review process and finalization of the manuscript. All authors read and approved the final manuscript

\section{Competing interests}

The authors declare that they have no competing interests.

\section{Consent for publication}

Not applicable.

\section{Ethics approval}

Mouse husbandry and procedures were performed under the guidelines and protocols of the Institutional Animal Care Use Committee (IACUC), the Division of Animals Resources (DAR) of The Scripps Research Institute and by the Institutional Animal Care and Use Committee of Nippon Medical School and the National Institute of Health Guide for the Care and Use of Laboratory Animals (Japan).

\section{Publisher's Note}

Springer Nature remains neutral with regard to jurisdictional claims in published maps and institutional affiliations.

\section{Author details}

'Department of Molecular Medicine, The Scripps Research Institute, $10550 \mathrm{~N}$. Torrey Pines Road, La Jolla, CA 92037, USA. ²Department of Physiology, Nippon Medical School, Tokyo 113-8602, Japan. ${ }^{3}$ Department of Infection and Immunity, Luxembourg Institute of Health, Esch-sur-Alzette L-4354, Luxembourg. ${ }^{4}$ Department of Neuroscience, The Scripps Research Institute, 10550 N. Torrey Pines Road, La Jolla, CA 92037, USA. ${ }^{5}$ Dipartimento di Scienze Cliniche e Molecolari, Università Politecnica delle Marche, 60020 Ancona, Italy. ${ }^{6}$ Cellular Neurobiology Laboratory, Salk Institute for Biological Studies, La Jolla, CA 92307, USA. Dorris Neuroscience Center, The Scripps Research Institute, 10550 N. Torrey Pines Road, La Jolla, CA 92037, USA.

Received: 17 November 2016 Accepted: 7 April 2017

Published online: 21 April 2017

\section{References}

1. de Lau LM, Breteler MM. Epidemiology of Parkinson's disease. Lancet Neurol. 2006:5:525-35

2. Davie CA. A review of Parkinson's disease. Br Med Bull. 2008;86:109-27.

3. Sapolsky RM. Stress, glucocorticoids, and damage to the nervous system: the current state of confusion. Stress. 1996;1:1-19.

4. Sapolsky RM, Uno H, Rebert CS, Finch CE. Hippocampal damage associated with prolonged glucocorticoid exposure in primates. J Neurosci. 1990;10:2897-902.

5. Sanchez-Alavez M, Conti B, Moroncini G, Criado JR. Contributions of neuronal prion protein on sleep recovery and stress response following sleep deprivation. Brain Res. 2007;1158:71-80.

6. Gibberd FB, Simmonds JP. Neurological disease in ex-far-east prisoners of war. Lancet. 1980;2:135-7.

7. Hemmerle AM, Herman JP, Seroogy KB. Stress, depression and Parkinson's disease. Exp Neurol. 2012;233:79-86.

8. Smith AD, Castro SL, Zigmond MJ. Stress-induced Parkinson's disease: a working hypothesis. Physiol Behav. 2002;77:527-31. 
9. Smith LK, Jadavji NM, Colwell KL, Katrina Perehudoff S, Metz GA. Stress accelerates neural degeneration and exaggerates motor symptoms in a rat model of Parkinson's disease. Eur J Neurosci. 2008;27:2133-46.

10. Sugama S, Kakinuma Y. Loss of dopaminergic neurons occurs in the ventral tegmental area and hypothalamus of rats following chronic stress: possible pathogenetic loci for depression involved in Parkinson's disease. Neurosci Res. 2016;111:48-55

11. Sugama S, Sekiyama K, Kodama T, Takamatsu Y, Takenouchi T, Hashimoto M, Bruno C, Kakinuma Y. Chronic restraint stress triggers dopaminergic and noradrenergic neurodegeneration: possible role of chronic stress in the onset of Parkinson's disease. Brain Behav Immun. 2016;51:39-46.

12. Hemmerle AM, Dickerson JW, Herman JP, Seroogy KB. Stress exacerbates experimental Parkinson's disease. Mol Psychiatry. 2014;19:638-40.

13. de Pablos RM, Herrera AJ, Espinosa-Oliva AM, Sarmiento M, Munoz MF, Machado A, Venero JL. Chronic stress enhances microglia activation and exacerbates death of nigral dopaminergic neurons under conditions of inflammation. J Neuroinflammation. 2014;11:34.

14. Ong LK, Zhao Z, Kluge M, Tebay C, Zalewska K, Dickson PW, Johnson SJ, Nilsson M, Walker FR. Reconsidering the role of glial cells in chronic stressinduced dopaminergic neurons loss within the substantia nigra? Friend or foe? Brain Behav Immun. 2017;60:117-25.

15. Sugama S, Fujita M, Hashimoto M, Conti B. Stress induced morphological microglial activation in the rodent brain: involvement of interleukin-18. Neuroscience. 2007;146:1388-99.

16. Hinwood M, Morandini J, Day TA, Walker FR. Evidence that microglia mediate the neurobiological effects of chronic psychological stress on the medial prefrontal cortex. Cereb Cortex. 2012;22:1442-54.

17. Tynan RJ, Naicker S, Hinwood M, Nalivaiko E, Buller KM, Pow DV, Day TA, Walker FR. Chronic stress alters the density and morphology of microglia in a subset of stress-responsive brain regions. Brain Behav Immun. 2010;24:1058-68.

18. Sugama S, Takenouchi T, Fujita M, Conti B, Hashimoto M. Differential microglial activation between acute stress and lipopolysaccharide treatment. J Neuroimmunol. 2009;207:24-31.

19. Diz-Chaves Y, Pernia O, Carrero P, Garcia-Segura LM. Prenatal stress causes alterations in the morphology of microglia and the inflammatory response of the hippocampus of adult female mice. J Neuroinflammation. 2012;9:71.

20. Kojo A, Yamada K, Kubo KY, Yamashita A, Yamamoto T. Occlusal disharmony in mice transiently activates microglia in hippocampal CA1 region but not in dentate gyrus. Tohoku J Exp Med. 2010;221:237-43.

21. Sugama S, Takenouchi T, Fujita M, Kitani H, Hashimoto M. Cold stress induced morphological microglial activation and increased IL-1beta expression in astroglial cells in rat brain. J Neuroimmunol. 2011;233:29-36.

22. Mcgeer PL, Itagaki S, Akiyama H, Mcgeer EG. Rate of cell death in Parkinsonism indicates active neuropathological process. Ann Neurol. 1988;24:574-6.

23. Perez Nievas BG, Hammerschmidt T, Kummer MP, Terwel D, Leza JC, Heneka MT. Restraint stress increases neuroinflammation independently of amyloid beta levels in amyloid precursor protein/PS1 transgenic mice. J Neurochem. 2011;116:43-52.

24. Glass CK, Saijo K, Winner B, Marchetto MC, Gage FH. Mechanisms underlying inflammation in neurodegeneration. Cell. 2010;140:918-34.

25. Tansey MG, Goldberg MS. Neuroinflammation in Parkinson's disease: its role in neuronal death and implications for therapeutic intervention. Neurobiol Dis. 2010;37:510-8.

26. Morrison BE, Marcondes MC, Nomura DK, Sanchez-Alavez M, Sanchez-Gonzalez A, Saar I, Kim KS, Bartfai T, Maher P, Sugama S, Conti B. Cutting edge: IL-13ralpha1 expression in dopaminergic neurons contributes to their oxidative stress-mediated loss following chronic peripheral treatment with lipopolysaccharide. J Immunol. 2012;189:5498-502.

27. Mori S, Maher P, Conti B. Neuroimmunology of the interleukins 13 and 4. Brain Sci. 2016;6(2):18.

28. Ramalingam TR, Pesce JT, Sheikh F, Cheever AW, Mentink-Kane MM, Wilson MS, Stevens S, Valenzuela DM, Murphy AJ, Yancopoulos GD, et al. Unique functions of the type II interleukin 4 receptor identified in mice lacking the interleukin 13 receptor alpha1 chain. Nat Immunol. 2008;9:25-33.

29. Volpe BT, Blau AD, Wessel TC, Saji M. Delayed histopathological neurona damage in the substantia nigra compacta (nucleus A9) after transient forebrain ischaemia. Neurobiol Dis. 1995;2:119-27.

30. Volpe BT, Wildmann J, Altar CA. Brain-derived neurotrophic factor prevents the loss of nigral neurons induced by excitotoxic striatal-pallidal lesions. Neuroscience. 1998;83:741-8.
31. Yu JT, Lee CH, Yoo KY, Choi JH, Li H, Park OK, Yan B, Hwang IK, Kwon YG, Kim YM, Won MH. Maintenance of anti-inflammatory cytokines and reduction of glial activation in the ischemic hippocampal CA1 region preconditioned with lipopolysaccharide. J Neurol Sci. 2010;296:69-78.

32. Zhao X, Wang H, Sun G, Zhang J, Edwards NJ, Aronowski J. Neuronal interleukin-4 as a modulator of microglial pathways and ischemic brain damage. J Neurosci. 2015;35:11281-91.

33. Brombacher TM, Nono JK, De Gouveia KS, Makena N, Darby M, Womersley J, Tamgue O, Brombacher F. IL-13-mediated regulation of learning and memory. J Immunol. 2017;198(7):2681-8.

34. Fontella FU, Cimarosti H, Crema LM, Thomazi AP, Leite MC, Salbego C, Goncalves CA, Wofchuk S, Dalmaz C, Netto CA. Acute and repeated restraint stress influences cellular damage in rat hippocampal slices exposed to oxygen and glucose deprivation. Brain Res Bull. 2005;65:443-50.

35. Fontella FU, Siqueira IR, Vasconcellos AP, Tabajara AS, Netto CA, Dalmaz C. Repeated restraint stress induces oxidative damage in rat hippocampus. Neurochem Res. 2005;30:105-11.

36. Kreisel T, Frank MG, Licht T, Reshef R, Ben-Menachem-Zidon O, Baratta MV, Maier SF, Yirmiya R. Dynamic microglial alterations underlie stress-induced depressive-like behavior and suppressed neurogenesis. Mol Psychiatry. 2014;19:699-709.

37. Bollinger JL, Bergeon Burns CM, Wellman CL. Differential effects of stress on microglial cell activation in male and female medial prefrontal cortex. Brain Behav Immun. 2016;52:88-97.

38. Rasheed N, Ahmad A, Pandey CP, Chaturvedi RK, Lohani M, Palit G. Differential response of central dopaminergic system in acute and chronic unpredictable stress models in rats. Neurochem Res. 2010;35:22-32.

39. Mizoguchi K, Yuzurihara M, Ishige A, Sasaki H, Chui DH, Tabira T. Chronic stress induces impairment of spatial working memory because of prefrontal dopaminergic dysfunction. J Neurosci. 2000;20:1568-74.

40. Watanabe Y, Gould E, McEwen BS. Stress induces atrophy of apical dendrites of hippocampal CA3 pyramidal neurons. Brain Res. 1992;588:341-5.

41. Monteiro S, Roque S, de Sa-Calcada D, Sousa N, Correia-Neves M, Cerqueira JJ. An efficient chronic unpredictable stress protocol to induce stress-related responses in C57BL/6 mice. Front Psych. 2015;6:6.

42. Bardin L, Malfetes N, Newman-Tancredi A, Depoortere R. Chronic restraint stress induces mechanical and cold allodynia, and enhances inflammatory pain in rat: relevance to human stress-associated painful pathologies. Behav Brain Res. 2009;205:360-6.

43. Voorhees JL, Tarr AJ, Wohleb ES, Godbout JP, Mo X, Sheridan JF, Eubank TD, Marsh CB. Prolonged restraint stress increases $\mathrm{IL}-6$, reduces $\mathrm{IL}-10$, and causes persistent depressive-like behavior that is reversed by recombinant IL-10. Plos One. 2013;8:e58488.

44. Conti B, Sanchez-Alavez M, Winsky-Sommerer R, Morale MC, Lucero J, Brownell S, Fabre V, Huitron-Resendiz S, Henriksen S, Zorrilla EP, et al. Transgenic mice with a reduced core body temperature have an increased life span. Science. 2006;314:825-8.

\section{Submit your next manuscript to BioMed Central and we will help you at every step:}

- We accept pre-submission inquiries

- Our selector tool helps you to find the most relevant journal

- We provide round the clock customer support

- Convenient online submission

- Thorough peer review

- Inclusion in PubMed and all major indexing services

- Maximum visibility for your research

Submit your manuscript at www.biomedcentral.com/submit
) Biomed Central 